\title{
ON A FAST DIFFUSION EQUATION WITH SOURCE
}

\author{
JONG-SHENQ GUO AND YUNG-JEN L. GuO ${ }^{\dagger}$
}

(Received January 19, 2000, revised December 4, 2000)

\begin{abstract}
We study in this paper the positive solution of the Cauchy problem for a fast diffusion equation with source. We derive a secondary critical exponent of the behavior of the initial value at infinity for the existence of global (in time) and nonglobal solutions of the Cauchy problem. Furthermore, the large time behaviors of those global solutions are also studied.
\end{abstract}

1. Introduction. In this paper, we study the positive solution of the Cauchy problem for the equation

$$
u_{t}=\Delta\left(u^{m}\right)+u^{p}, \quad x \in \boldsymbol{R}^{n}, t>0,
$$

with the initial condition

$$
u(x, 0)=u_{0}(x), \quad x \in \boldsymbol{R}^{n},
$$

where $m>0, p>1$, and $u_{0}$ is a bounded positive continuous function in $\boldsymbol{R}^{n}$.

For $m=1$, the equation (1.1) is the standard heat equation with a source term. A solution is said to blow up in finite time if its sup norm tends to infinity in finite time. In a paper by Fujita [5], it is shown that there is a critical exponent $p_{1}^{*}=1+2 / n$ such that the solution $u$ of (1.1)-(1.2) blows up in finite time for all $u_{0}$, if $1<p<p_{1}^{*}$; and there are global (in time) solutions and nonglobal (i.e., blowing-up in finite time) solutions, if $p>p_{1}^{*}$. This value $p_{1}^{*}$ is the so-called Fujita exponent. In fact, the Fujita exponent for (1.1)-(1.2) for any $m>0$ is given by $p_{m}^{*}=m+2 / n$. For more references on this topic, we refer the readers to two nice survey papers [11] and [1].

Recently, Mukai, Mochizuki, and Huang [12] have studied the case when $1<m<p$. It is shown, among other things, that for $p>p_{m}^{*}$ there is a secondary critical exponent $a^{*}=2 /(p-m)$ such that the solution $u$ of (1.1)-(1.2) blows up in finite time for any initial value $u_{0}$ which behaves like $|x|^{-a}$ at $|x|=\infty$, if $a \in\left(0, a^{*}\right)$; and there are global solutions for initial value $u_{0}$ which behaves like $|x|^{-a}$ at $|x|=\infty$, if $a \in\left(a^{*}, n\right)$. Motivated by their work, we shall extend these results to the case when $(1-2 / n)_{+}<m<1$ and $p>p_{m}^{*}$.

We also study the large time behaviors of global solutions of the Cauchy problem (1.1)(1.2) for the case $m<1$. The case $m>1$ is treated in [12]. For more references on the large

2000 Mathematics Subject Classification. Primary 35K55; Secondary 35K57, 34B15.

Key words and phrases. Fast diffusion equation, source, critical exponent.

${ }^{\dagger}$ This work was partially supported by National Science Council of the Republic of China under the contracts NSC 88-2115-M-003-007 and NSC 88-2115-M-003-002. 
time behaviors for various problems, we refer the readers to the references cited in the paper [6].

This paper is organized as follows. First, some preliminaries are given in Section 2. We study the forward self-similar solutions of the equation (1.1) without the source term $u^{p}$. Also, we prove a comparison principle. In Section 3, we derive the secondary exponent which is given by $a^{*}=2 /(p-m)$. Finally, we study the large time behaviors of global solutions. It is shown that as $t \rightarrow \infty$ the solution $u$ of (1.1)-(1.2) behaves like the self-similar solutions which are derived in Section 2.

2. Preliminaries. In the sequel, we always assume that $(1-2 / n)_{+}<m<1$ and $p>m+2 / n$. Set $l=2 /(1-m)$. Note that $n<l$. First, we recall from [9] that for any $a \in(0, n)$ and any $M>0$ there is a unique positive global solution $U_{M, a}(x, t)$ to the Cauchy problem

$$
\begin{gathered}
u_{t}=\Delta\left(u^{m}\right), \quad x \in \boldsymbol{R}^{n}, t>0, \\
u(x, 0)=M|x|^{-a}, \quad x \in \boldsymbol{R}^{n} .
\end{gathered}
$$

Here the initial function $M|x|^{-a}$ is locally integrable in $\boldsymbol{R}^{n}$, since $a \in(0, n)$. Indeed, this solution is the so-called forward self-similar solution of (2.1) given by

$$
U_{M, a}(x, t)=t^{-\beta a} g_{M}\left(\frac{|x|}{t^{\beta}}\right), \quad \beta=\frac{1}{2-(1-m) a},
$$

for some positive function $g_{M}$ satisfying the following boundary value problem:

$$
\begin{gathered}
\left(g^{m}\right)^{\prime \prime}+\frac{n-1}{\xi}\left(g^{m}\right)^{\prime}+\beta a g+\beta \xi g^{\prime}=0, \quad \xi>0, \\
g^{\prime}(0)=0, \quad \lim _{\xi \rightarrow \infty} \xi^{a} g(\xi)=M .
\end{gathered}
$$

Note that $\beta>0$, since $a<n<l$.

We remark that the existence and uniqueness of the self-similar solution can also be derived by the following ordinary differential equation approach (cf. [7]). In this approach, we can derive more properties of the solutions. We shall outline the main idea as follows.

Given a fixed $\eta>0$. We consider the following initial value problem:

$$
\begin{gathered}
h^{\prime \prime}+\frac{n-1}{\xi} h^{\prime}+\beta a h^{q}+\beta \xi\left(h^{q}\right)^{\prime}=0, \quad \xi>0, \\
h^{\prime}(0)=0, \quad h(0)=\eta,
\end{gathered}
$$

where $q=1 / m$. The existence and uniqueness of local solution $h=h_{\eta}$ of (2.6)-(2.7) follows from the standard theory of ordinary differential equations. Define

$$
\rho(y)=\exp \left\{\beta q \int_{0}^{y} \xi h(\xi)^{q-1} d \xi\right\} .
$$


Then (2.6) can be rewritten as

$$
h^{\prime}(\xi)=-\frac{\beta a}{\xi^{n-1} \rho(\xi)} \int_{0}^{\xi} s^{n-1} \rho(s) h(s)^{q} d s, \quad \xi>0 .
$$

Hence $h$ is decreasing as long as $h$ is positive.

We claim that $h>0$ for all $\xi>0$. Suppose for the contradiction that $h>0$ in $[0, R)$ and $h(R)=0$ for some $R>0$. Set

$$
H(\xi)=\frac{1}{2}\left[h^{\prime}(\xi)\right]^{2}+\beta a \int_{0}^{h(\xi)} s^{q} d s .
$$

Since $H^{\prime}(\xi) \leq 0$ for $\xi \in[0, R)$, we obtain that $h^{\prime}(\xi)$ is bounded for $\xi \in[0, R)$. Now, multiplying the equation (2.6) by $\xi^{n-1}$ and integrating it over [0,y] for $y<R$, we obtain that

$$
\beta y^{n} h(y)^{q}+\beta(a-n) \int_{0}^{y} \xi^{n-1} h^{q}(\xi) d \xi+y^{n-1} h^{\prime}(y)=0 .
$$

Letting $y \rightarrow R^{-}$and noting $h^{\prime}<0$ in $(0, R)$, we reach a contradiction, since $a<n$. Therefore, the local solution can be extended to a global solution.

Next, by applying a method used in [7], we can show that $h(\xi) \rightarrow 0$ as $\xi \rightarrow \infty$. Moreover,

$$
\lim _{\xi \rightarrow \infty} \xi^{a} h^{q}(\xi)=M
$$

for some $M=M(\eta)>0$. It remains to show that there is a one-to-one correspondence between $M \in(0, \infty)$ and $\eta \in(0, \infty)$. Indeed, this can be seen from the relation

$$
h_{\eta}(\xi)=\eta h_{1}\left(\eta^{\sigma} \xi\right), \sigma=\frac{1-m}{2 m}>0
$$

Hence

$$
M(\eta)=\eta^{1 /(2 \beta m)} M(1) .
$$

We conclude that for each $M>0$ there is a unique positive solution $g_{M}$ satisfying (2.4)-(2.5).

We also need a comparison principle which is similar to Proposition 2.1 in [6].

Let $\phi \in C_{0}^{\infty}\left(\boldsymbol{R}^{n}\right)$ be fixed such that $0 \leq \phi \leq 1, \phi \equiv 1$ for $|x| \leq 1, \phi \equiv 0$ for $|x| \geq 2$, and

$$
E(\phi)=\left(\int_{\mathbf{R}^{n}}|\Delta \phi|^{1 /(1-m)} \phi^{-m /(1-m)} d x\right)^{1-m}<\infty .
$$

For the existence of such function $\phi$, we refer the readers to [9] or [8, p. 1356].

For this $\phi$ and for any $R>0$, let $\phi_{R}(x)=\phi(x / R)$. Notice that

$$
E\left(\phi_{R}\right)=R^{-2+n(1-m)} E(\phi) \text {. }
$$

Denote

$$
\mathcal{L} u=u_{t}-\Delta\left(u^{m}\right)-u^{p} .
$$

For any $R>0$, let $B_{R}=\left\{x \in \boldsymbol{R}^{n}|| x \mid<R\right\}$. We now prove the following comparison principle. 
Proposition 2.1. Let $u, v \in C^{\infty}\left(\boldsymbol{R}^{n} \times(0, T]\right) \cap C^{0}\left(\boldsymbol{R}^{n} \times[0, T]\right)$ and $0<u, v \leq M$ in $\boldsymbol{R}^{n} \times[0, T]$ for some $M>0$ and $T>0$. Suppose that $\mathcal{L} u \leq \mathcal{L} v$ in $\boldsymbol{R}^{n} \times(0, T]$ in the classical sense and $u(x, 0) \leq v(x, 0)$ for $x \in \boldsymbol{R}^{n}$. Then $u \leq v$ in $\boldsymbol{R}^{n} \times(0, T]$.

PROOF. Let $g$ be the characteristic function of the set $\{u>v\}$. It follows from Kato's inequality [10] that

$$
\Delta\left[\left(u^{m}-v^{m}\right)_{+}\right] \geq g \Delta\left(u^{m}-v^{m}\right),
$$

where $z_{+}=\max (z, 0)$. Since $\mathcal{L} u \leq \mathcal{L} v$ and

$$
g\left(u^{p}-v^{p}\right) \leq p M^{p-1}(u-v)_{+},
$$

it follows from (2.14) that

$$
\frac{\partial}{\partial t}\left[(u-v)_{+}\right] \leq \Delta\left[\left(u^{m}-v^{m}\right)_{+}\right]+p M^{p-1}(u-v)_{+} .
$$

Multiplying (2.15) by $\phi_{R}$ and integrating it over $\boldsymbol{R}^{n}$, we get

$$
\begin{aligned}
\frac{\partial}{\partial t} & {\left[\int_{\boldsymbol{R}^{n}}(u-v)_{+} \phi_{R} d x\right] } \\
& \leq \int_{\boldsymbol{R}^{n}}\left(u^{m}-v^{m}\right)_{+}\left|\Delta \phi_{R}\right| d x+p M^{p-1} \int_{\boldsymbol{R}^{n}}(u-v)_{+} \phi_{R} d x \\
& \leq \int_{\boldsymbol{R}^{n}}\left[(u-v)_{+}\right]^{m}\left|\Delta \phi_{R}\right| d x+p M^{p-1} \int_{\boldsymbol{R}^{n}}(u-v)_{+} \phi_{R} d x \\
\leq & C R^{-2+n(1-m)}\left[\int_{\boldsymbol{R}^{n}}(u-v)_{+} \phi_{R} d x\right]^{m} \\
& +p M^{p-1} \int_{\boldsymbol{R}^{n}}(u-v)_{+} \phi_{R} d x
\end{aligned}
$$

for any $R>0$, where $C$ is a universal constant.

Let $L=p M^{p-1}$ and set

$$
h(t)=\int_{\mathbf{R}^{n}}(u-v)_{+}(x, t) \phi_{R}(x) d x .
$$

Then by (2.16) we have

$$
h^{\prime}(t)-L h(t) \leq C R^{-2+n(1-m)} h^{m}(t)
$$

and so

$$
\left(e^{-L t} h(t)\right)^{\prime} \leq C R^{-2+n(1-m)} e^{-L t} h^{m}(t) \leq C R^{-2+n(1-m)}\left[e^{-L t} h(t)\right]^{m} .
$$

By integrating the above inequality from 0 to $t$, we end up with

$$
\int_{\mathbf{R}^{n}}(u-v)_{+}(x, t) \phi_{R}(x) d x \leq C R^{-2 /(1-m)+n} t^{1 /(1-m)} e^{L t} .
$$

Hence we obtain that

$$
\int_{B_{R}}(u-v)_{+}(x, t) d x \leq C R^{-2 /(1-m)+n} t^{1 /(1-m)} e^{L t} .
$$


Since $2 /(1-m)>n$, by letting $R \rightarrow \infty$ in (2.17), we obtain that

$$
\int_{\mathbf{R}^{n}}(u-v)_{+}(x, t) d x=0 .
$$

This completes the proof of the proposition.

We remark here that any positive (classical) solution of (1.1) for $0<t \leq T$ is in $C^{\infty}\left(\boldsymbol{R}^{n} \times(0, T]\right)$. Indeed, this follows from a bootstrap argument by applying the standard differentiability theory of parabolic equations (cf. for example [3, p. 74]). Notice that $u>0$ and so $u$ has a positive lower bound in any compact subdomain of $\boldsymbol{R}^{n} \times(0, T]$. Furthermore, if $u_{0}$ is continuous, then the solution $u$ of (1.1)-(1.2) is continuous up to $t=0$, i.e., $u \in C^{0}\left(\boldsymbol{R}^{n} \times[0, T]\right)$. This can be seen by a regular approximation procedure and the standard regularity theory of parabolic equation.

3. Secondary critical exponent. Given $a>0$, we define

$$
\begin{gathered}
\Phi_{a}=\left\{\left.\phi \in B\left(\boldsymbol{R}^{n}\right)\left|\liminf _{|x| \rightarrow \infty}\right| x\right|^{a} \phi(x)>0\right\}, \\
\Phi^{a}=\left\{\left.\phi \in B\left(\boldsymbol{R}^{n}\right)\left|\limsup _{|x| \rightarrow \infty}\right| x\right|^{a} \phi(x)<\infty\right\},
\end{gathered}
$$

where $B\left(\boldsymbol{R}^{n}\right)$ denotes the space of bounded positive continuous functions in $\boldsymbol{R}^{n}$. Let $a^{*}=$ $2 /(p-m)$. Note that $p>m+2 / n$ implies that $0<a^{*}<n$.

First, we derive a blow-up result as follows.

THEOREM 3.1. Suppose that $u_{0} \in \Phi_{a}$ for some $a \in\left(0, a^{*}\right)$. Then the solution $u$ of the Cauchy problem (1.1)-(1.2) blows up in finite time.

Proof. Multiplying the equation (1.1) by $\phi_{\varepsilon}(x)=A e^{-\varepsilon|x|^{2}}$, where $\varepsilon>0$ and $A=$ $A(\varepsilon)=(\sqrt{\varepsilon /(4 \pi)})^{n}$, and integrating it over $\boldsymbol{R}^{n}$, we obtain

$$
\frac{d}{d t} \int_{\boldsymbol{R}^{n}} u(x, t) \phi_{\varepsilon}(x) d x=\int_{\boldsymbol{R}^{n}} \Delta\left(u^{m}\right)(x, t) \phi_{\varepsilon}(x) d x+\int_{\boldsymbol{R}^{n}} u^{p}(x, t) \phi_{\varepsilon}(x) d x .
$$

Since

$$
\int_{\boldsymbol{R}^{n}} \Delta\left(u^{m}\right)(x, t) \phi_{\varepsilon}(x) d x=\int_{\boldsymbol{R}^{n}} u^{m}(x, t) \Delta \phi_{\varepsilon}(x) d x \geq-2 \varepsilon n \int_{\boldsymbol{R}^{n}} u^{m}(x, t) \phi_{\varepsilon}(x) d x,
$$

and by Jensen's inequality

we derive that

$$
\begin{aligned}
\int_{\boldsymbol{R}^{n}} u^{p}(x, t) \phi_{\varepsilon}(x) d x & \geq\left(\int_{\boldsymbol{R}^{n}} u(x, t) \phi_{\varepsilon}(x) d x\right)^{p}, \\
\int_{\boldsymbol{R}^{n}} u^{m}(x, t) \phi_{\varepsilon}(x) d x & \leq\left(\int_{\boldsymbol{R}^{n}} u(x, t) \phi_{\varepsilon}(x) d x\right)^{m},
\end{aligned}
$$

$$
h^{\prime}(t) \geq h^{p}(t)-2 \varepsilon n h^{m}(t), t>0
$$

where

$$
h(t)=\int_{\boldsymbol{R}^{n}} u(x, t) \phi_{\varepsilon}(x) d x .
$$


If

$$
h(0)>(2 \varepsilon n)^{1 /(p-m)},
$$

then by (3.1) $h$ blows up in finite time, since $p>1$. Therefore, the solution $u$ blows up in finite time.

It remains to verify the condition (3.2). Since $u_{0} \in \Phi_{a}$ for some $a \in\left(0, a^{*}\right)$, there are positive constants $M$ and $R$ such that $u_{0}(x) \geq M|x|^{-a}$ for any $|x| \geq R$. We compute that

$$
\begin{aligned}
h(0) & =\int_{R^{n}} u_{0}(x) \phi_{\varepsilon}(x) d x \geq M A \int_{|x| \geq R}|x|^{-a} e^{-\varepsilon|x|^{2}} d x \\
& =M(\sqrt{4 \pi})^{-n} \varepsilon^{a / 2} \int_{|y| \geq R \sqrt{\varepsilon}}|y|^{-a} e^{-|y|^{2}} d y .
\end{aligned}
$$

Since $a<a^{*}$, we may choose $\varepsilon>0$ so small that (3.2) holds. Hence the theorem is proved.

We shall show that the exponent $a^{*}$ gives the secondary critical exponent for the existence of global and nonglobal solutions of the Cauchy problem (1.1)-(1.2).

Suppose that $\phi \in \Phi^{a}$ for some $a \in\left(a^{*}, n\right)$. Since $\phi \in \Phi^{a}$, there is a positive constant $K$ such that

$$
\phi(x) \leq K(1+|x|)^{-a} \text { for all } x \in \boldsymbol{R}^{n} .
$$

Choose $M>K$. Consider the self-similar solution

$$
U_{M, a}(x, t)=t^{-\beta a} g_{M}\left(\frac{|x|}{t^{\beta}}\right), \quad \beta=\frac{1}{2-(1-m) a} .
$$

Since

$$
\lim _{\xi \rightarrow \infty} \xi^{a} g_{M}(\xi)=M>K,
$$

there is a positive constant $R$ such that

$$
\xi^{a} g_{M}(\xi)>K \text { for any } \xi \geq R \text {. }
$$

Let $\gamma=g_{M}(R)$. Note that $g_{M}(R)=\min \left\{g_{M}(\xi) \mid \xi \in[0, R]\right\}>0$. Choose $\tau \in(0,1)$ such that $\tau^{-\beta a} \gamma>\|\phi\|_{\infty}$. Then it is easy to verify that $\phi(x) \leq U_{M, a}(x, \tau)$ for all $x \in \boldsymbol{R}^{n}$.

Let $\lambda>0$. Then $w(x, t)=\lambda U_{M, a}\left(x, \lambda^{m-1} t+\tau\right)$ is the solution of the problem

$$
\begin{gathered}
w_{t}=\Delta\left(w^{m}\right), t>0, x \in \boldsymbol{R}^{n}, \\
w(x, 0)=\lambda U_{M, a}(x, \tau), x \in \boldsymbol{R}^{n} .
\end{gathered}
$$

Let $\eta=g_{M}(0)$. Then

$$
\|w(\cdot, t)\|_{\infty}=\eta \lambda\left(\lambda^{m-1} t+\tau\right)^{-\beta a} .
$$

Introduce the function $v(x, t)=A(t) w(x, B(t))$, where $A(t)$ and $B(t)$ are solutions of the following problems:

$$
\begin{gathered}
A^{\prime}(t)=\eta^{p-1} \lambda^{p-1}\left[\lambda^{m-1} B(t)+\tau\right]^{-\beta a(p-1)} A^{p}(t), t>0 ; A(0)=1, \\
B^{\prime}(t)=A^{m-1}(t), t>0 ; B(0)=0 .
\end{gathered}
$$


PROPOSITION 3.2. There is a positive constant $\lambda_{0}=\lambda_{0}(\phi)$ such that the problem (3.4)-(3.5) has a global solution $(A(t), B(t))$ with $A(t)$ bounded in $[0, \infty)$, if $\lambda \in\left(0, \lambda_{0}\right)$.

Proof. Let $q=\beta a(p-1)-1$. Note that $q>0$, since $a \in\left(a^{*}, n\right)$. Let $\lambda_{0}$ be a positive constant defined by

$$
\left(\frac{p-m}{1-m}\right)\left(\frac{1-m}{q} \eta^{p-1} \lambda_{0}^{p-m} \tau^{-q}\right)^{(p-1) /(p-m)}=1 .
$$

Given a fixed $\lambda \in\left(0, \lambda_{0}\right)$, we define

$$
\begin{gathered}
D=D(\lambda)=\frac{1}{q} \eta^{p-1} \lambda^{p-m} \tau^{-q}, \\
A_{\infty}=A_{\infty}(\lambda)=[(1-m) D]^{1 /(m-p)}, \\
g(x)=g_{\lambda}(x)=1-\left[(p-1) D x^{1-m}+x^{1-p}\right], x \geq 1 .
\end{gathered}
$$

Note that $D>0$ and $A_{\infty} \in(1, \infty)$. Moreover, $g$ is continuous on $[1, \infty)$ such that $g(1)<0$, $g(+\infty)=-\infty$, and $\max _{x \geq 1} g(x)=g\left(A_{\infty}\right)>0$, since $0<\lambda<\lambda_{0}$.

The local existence and uniqueness of solution $(A(t), B(t))$ of (3.4)-(3.5) follows from the standard theory of initial value problem. We have $A^{\prime}(t)>0$ and $A(t)>1$ for $t>0$ as long as the solution exists. Notice that the solution of (3.4)-(3.5) can be continued as long as $A(t)$ is finite. Also, $B(t)$ is uniquely defined by

$$
B(t)=\int_{0}^{t} A^{m-1}(s) d s
$$

when $A(t)$ exists in $[0, t]$.

From (3.4), it follows that

$$
1-A^{1-p}(t)=(p-1) \eta^{p-1} \lambda^{p-1} \int_{0}^{t}\left[\lambda^{m-1} B(s)+\tau\right]^{-\beta a(p-1)} d s .
$$

Since

we obtain that

$$
B(s)=\int_{0}^{s} A^{m-1}(y) d y \geq A^{m-1}(t) s \text { for any } s \in[0, t],
$$

$$
1-A^{1-p}(t) \leq(p-1) D A^{1-m}(t) .
$$

Hence $g(A(t)) \leq 0$ as long as $A(t)$ exists. By the properties of $g$ and $A, A(t)<A_{\infty}$ as long as $A(t)$ exists. Otherwise, if $A(t) \geq A_{\infty}$ for some $t$, then there is $s \leq t$ such that $A(s)=A_{\infty}$ and so $g(A(s))>0$, a contradiction. Therefore, the solution exists for all $t \geq 0$ and $A(t)$ is bounded by $A_{\infty}$. The proof is completed.

We are ready to prove the following theorem.

THEOREM 3.3. Suppose that $u_{0}=\lambda \phi$ for some $\lambda>0$ and $\phi \in \Phi^{a}$ for some $a \in$ $\left(a^{*}, n\right)$. Then there is $\lambda_{0}=\lambda_{0}(\phi)>0$ such that the solution $u$ of the Cauchy problem (1.1)-(1.2) exists for all $t>0$, if $\lambda<\lambda_{0}$. 
Proof. Let $\lambda_{0}=\lambda_{0}(\phi)$ be the positive constant in Proposition 3.2. Define $v(x, t)=$ $A(t) w(x, B(t))$, where $(A(t), B(t))$ is the solution of the problem (3.4)-(3.5). Then it is easy to check that $v$ satisfies

$$
\begin{gathered}
v_{t} \geq \Delta\left(v^{m}\right)+v^{p}, \quad t>0, x \in \boldsymbol{R}^{n}, \\
v(x, 0)=w(x, 0)=\lambda U_{M, a}(x, \tau) \geq \lambda \phi(x), \quad x \in \boldsymbol{R}^{n} .
\end{gathered}
$$

Hence by Proposition 2.1 the solution $u$ of the Cauchy problem (1.1)-(1.2) with $u_{0}=\lambda \phi$ exists globally and $u \leq v$ in $\boldsymbol{R}^{n} \times[0, \infty)$, if $\lambda<\lambda_{0}$.

We remark that by (3.3) there is a positive constant $C$ such that

$$
\|u(\cdot, t)\|_{\infty} \leq C t^{-\beta a} \text { for all } t>0,
$$

for the solution $u$ in Theorem 3.3, since $A_{\infty}^{m-1} t \leq B(t) \leq t$.

4. Large time behavior. In this section, we shall always assume that $a \in\left(a^{*}, n\right)$ and that

$$
\lim _{|x| \rightarrow \infty}|x|^{a} \phi(x)=M
$$

for some $M>0$. Recall from Theorem 3.3 that the solution $u$ of the Cauchy problem (1.1)(1.2) with $u_{0}=\lambda \phi$ exists globally in time, if $\lambda<\lambda_{0}$, where $\lambda_{0}=\lambda_{0}(\phi)>0$. We shall study the behavior of $u(x, t)$ as $t \rightarrow \infty$ in this section and obtain the following result. The idea of the proof is based on the well-known rescaling method of Friedman and Kamin [4]. As before, let $\beta=1 /[2-(1-m) a]>0$.

THEOREM 4.1 As $t \rightarrow \infty$, we have

$$
t^{\beta a}\left|u(x, t)-U_{\lambda M, a}(x, t)\right| \rightarrow 0
$$

uniformly on any compact set $\left\{(x, t)|| x \mid \leq C t^{\beta}\right\}$ for all $C>0$.

PROOF. For $\sigma>1$, let

$$
u_{\sigma}(x, t)=\sigma^{\beta a} u\left(\sigma^{\beta} x, \sigma t\right)
$$

Then $u_{\sigma}$ satisfies

$$
\begin{gathered}
\left(u_{\sigma}\right)_{t}=\Delta\left(u_{\sigma}^{m}\right)+\sigma^{-v} u_{\sigma}^{p}, \quad t>0, x \in \boldsymbol{R}^{n}, \\
u_{\sigma}(x, 0)=\sigma^{\beta a} u_{0}\left(\sigma^{\beta} x\right) \equiv u_{0 \sigma}(x), \quad x \in \boldsymbol{R}^{n},
\end{gathered}
$$

where $v=[(p-m) a-2] \beta>0$, since $a \in\left(a^{*}, n\right)$.

Recall (3.6). We have the estimate

$$
u_{\sigma}(x, t) \leq C t^{-\beta a} \text { for any } t>0, x \in \boldsymbol{R}^{n} \text { and } \sigma>1 .
$$

Hence $\left\{\sigma^{-v} u_{\sigma}^{p} \mid \sigma>1\right\}$ is uniformly bounded in any compact subset of $Q \equiv \boldsymbol{R}^{n} \times(0, \infty)$. Using the regularity theory of quasilinear parabolic equations (cf. [2] and [13]), $\left\{u_{\sigma}\right\}$ is equicontinuous on any compact subset of $Q$. Then by a diagonal process there is a subsequence $\left\{u_{\sigma_{k}}\right\}$ such that

$$
u_{\sigma_{k}} \rightarrow U \text { uniformly in any compact subset of } Q \text { as } k \rightarrow \infty
$$


for some $U \in C^{0}(Q)$. It is easy to verify that $U$ satisfies the equation

$$
U_{t}=\Delta\left(U^{m}\right) \quad \text { in } Q
$$

and $U(x, 0)=\lambda M|x|^{-a}, x \in \boldsymbol{R}^{n}$. Hence the uniqueness implies that

$$
U(x, t)=U_{\lambda M, a}(x, t)=t^{-\beta a} g\left(\frac{|x|}{t^{\beta}}\right),
$$

where $g$ is the solution of (2.4-5) with

$$
\lim _{\xi \rightarrow \infty} \xi^{a} g(\xi)=\lambda M .
$$

Indeed, (4.1) holds as $\sigma \rightarrow \infty$. In particular, we have

$$
\sigma^{\beta a} u\left(\sigma^{\beta} y, \sigma\right)=u_{\sigma}(y, 1) \rightarrow g(|y|)
$$

as $\sigma \rightarrow \infty$ uniformly on compact subsets of $\boldsymbol{R}^{n}$. Take $\sigma=t$ and set $x=\sigma^{\beta} y$. Then we obtain

$$
t^{\beta a}\left|u(x, t)-U_{\lambda M, a}(x, t)\right| \rightarrow 0
$$

uniformly on any compact set $\left\{(x, t)|| x \mid \leq C t^{\beta}\right\}$ for all $C>0$.

\section{REFERENCES}

[ 1 ] K. Deng And H. A. Levine, The role of critical exponents in blow-up theorems: the sequel, J. Math. Anal. Appl. 243 (2000), 85-126.

[ 2 ] E. DiBenedetTo, Continuity of weak solutions to a general porous medium equation, Indiana Univ. Math. J. 32 (1983), 83-118.

[ 3 ] A. Friedman, Partial Differential Equations of Parabolic Type, Prentice-Hall, Englewood Cliffs, N. J. 1964.

[ 4 ] A. FRIEDMAN AND S. KAMIN, The asymptotic behavior of gas in an $n$-dimensional porous medium, Trans. Amer. Math. Soc. 262 (1980), 551-563.

[ 5 ] H. Fujita, On the blowing up of solutions of the Cauchy problem for $u_{t}=\Delta u+u^{1+\alpha}$, J. Fac. Sci. Univ. Tokyo Sect. I 13 (1966), 109-124.

[ 6 ] J.-S. GuO, Large time behavior of solutions of a fast diffusion equation with source, Nonlinear Anal. 23 (1994) 1559-1568.

[ 7 ] J.-S. GuO, Similarity solutions for a quasilinear parabolic equation, J. Austral. Math. Soc. Ser. B 37 (1995), 253-266.

[ 8 ] J.-S. Guo, On the Cauchy problem for a very fast diffusion equation, Comm. Partial Differential. Equations 21 (1996), 1349-1365.

[ 9 ] M. A. Herrero And M. Pierre, The Cauchy problem for $u_{t}=\Delta u^{m}$ when $0<m<1$, Trans. Amer. Math. Soc. 291 (1985), 145-168.

[10] T. KATO, Schrödinger operators with singular potentials, Israel J. Math. 13 (1972), 135-148.

[11] H. A. LEVINE, The role of critical exponents in blowup theorems, SIAM Rev. 32 (1990), 262-288.

[12] K. Mukai, K. MochizuKi AND Q. HuANG, Large time behavior and life span for a quasilinear parabolic equation with slowly decaying initial values, Nonlinear Anal. 39 (2000), 33-45.

[13] P. E. SACKS, Continuity of solutions of a singular parabolic equation, Nonlinear Anal. 7 (1983), 387-409.

DEPARTMENT OF MATHEMATICS

NATIONAL TAIWAN NORMAL UNIVERSITY

88, SEC. 4, TING CHOU ROAD

TAIPEI 117

TAIWAN 